Please quote as: Neil, L.; Hansen, D.; Lindgaard, G.; Kogan, S.; Leimeister, J. M.; Oakley, K. \& Selker, T. (2006): SIG Online Health Communities. In: Proceedings of the Conference on Human Computer Interaction 2006 (CHI 2006), Montreal. 


\section{Online Health Communities}

\author{
Lisa Neal \\ eLearn Magazine \\ 3 Valley Road \\ Lexington, MA 02421 USA \\ lisa@acm.org \\ Gitte Lindgaard \\ Human Oriented Technology \\ Lab (HOTLab) \\ Carleton University \\ Ottawa \\ Ontario, K1S 5B6 Canada \\ Gitte_lindgaard@carleton.ca \\ Kate Oakley \\ Human Oriented Technology Lab Munich, Germany \\ (HOTLab), Dept. of Psychology leimeister@in.tum.de \\ Carleton University \\ Ottawa \\ Ontario, K1S 5B6 Canada \\ koakley@connect.carleton.ca \\ Ted Selker \\ MIT Media Lab \\ Wiesner Building, E15 \\ 20 Ames Street \\ Cambridge, MA 02139 USA \\ selker@media.mit.edu
}

Copyright is held by the author/owner(s).

CHI 2006, April 22-27, 2006, Montréal, Québec, Canada.

ACM 1-59593-298-4/06/0004.

\begin{abstract}
Online health communities provide a means for patients and their families to learn about an illness, seek and offer support, and connect with others in similar circumstances. They are supported by a variety of technologies (e.g., email lists, forums, chat rooms) and are hosted by patients, advocacy groups, medical organizations, and corporations. They raise difficult design challenges because of the wide variability of members' medical expertise, the severity of problems due to misinformation, and the need for emotional support. The importance of on-line health communities is evidenced by their popularity, as well as the significant impact they have on the lives of their members. This Special Interest Group (SIG) will explore current trends in online health communities, as well as discuss the socio-technical design challenges and opportunities that they afford.
\end{abstract}

\section{Keywords}

Online health communities, online communities, virtual communities, medicine, support groups, e-learning, health care 


\section{ACM Classification Keywords}

H5.3. Information Interfaces and Presentation: Group and Organization Interfaces---Asynchronous

interaction, Computer-supported cooperative work, Web-based interaction

\section{Introduction}

Changes in the health care system coupled with increased infiltration and use of the Internet have resulted in heavier reliance on the Internet for disease and health education. Existing medical websites, such as WebMD [3], have recognized that they will have more visitors if they offer interactive community features such as web forums and chat rooms. Unfortunately, in many cases these community features are designed by clinicians who do not have an in-depth understanding of how to design for usability and sociability [2], yet, the criticality of health-related information necessitates careful consideration of design and usability. Further, patients and their families may be under stress and the emotional burden necessitates more careful design and evaluation. Perhaps these challenges are part of the reason that researchers have not yet conclusively demonstrated the health benefits of online support communities [1].

Although little research has directly measured health benefits, it has pointed to several situations where online health communities aid patients. These benefits include enhanced health literacy, improved quality of life, better decision making, and patients who feel less alone and more empowered [4]. Many online health communities provide a holistic approach to meeting medical, social, and emotional needs; an approach that is difficult to receive through traditional medical consultations. The benefits of online health communities are especially appreciated by individuals with mobility challenges, potentially embarrassing medical conditions, or caretaker responsibilities that may prohibit them from participating in face-to-face medical and emotional support [4].

For these advantages to accrue, systems must be developed that are accessible, welcoming, easy to navigate and use, and able to help members discern information quality and interact with other participants in meaningful ways. We believe that the successful design of such systems will be facilitated by collaborations among health professionals, informed designers, and patients. Health professionals and patients can help explain the physical and emotional stages that individuals go through once they are diagnosed with a particular disease. Health professionals understand the risks of misinformation and the role that healthcare providers should play. Patients understand the practical information about coping with a disease and the importance of social support and empathy. Systems designers are needed to understand and explain the technological options available to online health communities and the implications of specific design choices.

We hope to bring together a variety of individuals with backgrounds in social science, design, and health for this SIG. Both researchers and practitioners are encouraged to participate, especially those working on online communities, although the appeal will be greatest to people working in or interested in health care. Some CHI attendees may be interested in the SIG due to personal experiences with the use of online communities. 


\section{SIG Agenda}

Before CHI2006, we will set up an online meeting place where potential SIG attendees can view and add online health communities of interest, and can discuss online health community issues and research. The SIG will of course be open to people who did not participate online prior to the SIG.

We will start by examining recent trends in online health communities. What technological capabilities and forms of participation are currently used and what best practices and novel approaches have been taken? We will also discuss the general market factors such as who is funding online health communities, what are their primary objectives (e.g., health behavior change, education, support), and if these differ for people with different diseases, ages, or cultures.

We will then move on to the design and evaluation of online health communities. We will select and cover the questions of greatest interest to attendees from the following list:

\section{General Questions}

- What are the unique characteristics of online health communities as opposed to other online communities?

- What is the role of online health communities in the larger medical arena?

- How can online health communities be used to attract initial participation and support ongoing participation? What marketing techniques are unique to the medical realm?

\section{Design Questions}

- How can varying levels of health literacy and knowledge be accommodated? What designs help increase comprehension and retention?

- How can interactivity with peers and experts be supported? What community roles (e.g., moderator, support provider, seminar presenter) should be encouraged and what technologies support those roles? What indicators of community participation levels are valuable to other participants?

- What social or technical mechanisms can help promote high quality information and demote low quality information? How should quality be determined?

- How can the interface and content be customized to account for differences in medical condition, emotional state, or other health related variables?

- What design choices should be made differently to meet the needs of different patient populations (i.e., those with different diseases, cultural backgrounds, or personal traits)? What are some good examples of such customization?

- How can technologies (e.g., presence awareness, emoticons) be used to increase connectedness and social support? What techniques can be used to convey subtle social cues in media-poor environments?

- What design innovations can help newcomers (who are often newly diagnosed individuals) become socially integrated into the community?

- How can online health communities more seamlessly integrate with existing medical journals, encyclopedias, and texts? 


\section{Evaluation Questions}

- What are the various objectives of online health communities and how can one determine if they are being met? What data would be required?

- What types of evaluation are most appropriate for measuring success (e.g., formative evaluation of usability, appeal, and effectiveness at achieving goals; summative evaluation; health related measures)? How and to whom should different types of evaluation data be communicated, including participants?

We will conclude the SIG with a discussion of a research agenda, focused on the unique contributions that $\mathrm{HCI}$ researchers and practitioners can provide to the area of online health communities. We will also discuss possible future collaborations and determine if there is interest in continuing to meet online or in person following the SIG. Furthermore, we will document and publish the results of the workshop for broader dissemination.

\section{Citations}

[1] Eysenbach, G, Powell, J. Englesakis, M., Rizo, C. and Stern, A. Health related virtual communities and electronic support groups: systematic review of the effects of online peer to peer interactions. BMJ, 328 (2004), 1166-1170.

[2] Preece, J. Online Communities: Designing Usability Supporting Sociability. John Wiley \& Sons, Chichester, UK, 2000.

[3] WebMD http://www.webmd.com

[4] White, M. and Dorman, S.M. Receiving social support online: implications for health education. Health Education Research, 16 No. 6 (2001), 693-707 